\title{
On the metrical theory of a non-regular continued fraction expansion
}

\author{
Dan Lascu, George Cîrlig
}

\begin{abstract}
We introduced a new continued fraction expansions in our previous paper. For these expansions, we show the Brodén-Borel-Lévy type formula. Furthermore, we compute the transition probability function from this and the symbolic dynamical system of the natural number with the unilateral shift.
\end{abstract}

\section{Introduction}

The purpose of this paper is to study the stochastic behavior of some continued fraction expansions. Furthermore, measure theoretical dynamical systems arising them are investigated. First we outline the historical framework of the continued fractions. Then, in Section 1.2, we present the current framework. In Sections 3 we give the basic metric properties of the non-regular continued fraction expansions. The main results will be shown in Section 4.

\subsection{Historical background}

To this day the Gauss map, on which metrical theory of regular continued fraction $(\mathrm{RCF})$ is based, has fascinated researchers from various branches of mathematics and science, with many applications in computer science, cosmology and chaos theory [4]. In the last century, mathematicians broke new

Key Words: continued fractions, incomplete quotients, invariant measure

2010 Mathematics Subject Classification: 11A55, 11K50, 11J70, 28D05, 40A15.

Received: October, 2013.

Revised: December, 2013.

Accepted: December, 2013. 
ground in this area. Apart from the RCF expansion, very many other continued fraction expansions were studied.

One of the first and still one of the most important results in the metrical theory of continued fractions is so-called Gauss-Kuzmin theorem. Write $x \in$ $[0,1)$ as a regular continued fraction

$$
x=\frac{1}{a_{1}+\frac{1}{a_{2}+\frac{1}{a_{3}+\ddots}}}:=\left[a_{1}, a_{2}, a_{3}, \ldots\right],
$$

with $a_{n} \in \mathbb{N}_{+}$, where $\mathbb{N}_{+}=\{1,2,3, \ldots\}$.

The metrical theory of continued fractions started on 25th October 1800, with a note by Gauss in his mathematical diary [3]. Gauss wrote that (in modern notation)

$$
\lim _{n \rightarrow \infty} \lambda\left(\tau^{n} \leq x\right)=\frac{\log (1+x)}{\log 2}, x \in I:=[0,1] .
$$

Here $\lambda$ is Lebesgue measure and the map $\tau:[0,1) \rightarrow[0,1)$, the so-called regular continued fraction (or Gauss) map, is defined by

$$
\tau(x):=\frac{1}{x}-\left\lfloor\frac{1}{x}\right\rfloor, \quad x \neq 0 ; \tau(0):=0,
$$

where $\lfloor\cdot\rfloor$ denotes the floor (or entire) function. Gauss' proof (if any) has never been found. A little more than 11 years later, in a letter dated 30 January 1812, Gauss asked Laplace to estimate the error

$$
e_{n}(x):=\lambda\left(\tau^{-n}[0, x]\right)-\frac{\log (1+x)}{\log 2}, \quad n \geq 1, x \in I .
$$

This has been called Gauss' Problem. It received a first solution more than a century later, when R.O. Kuzmin [8] showed in 1928 that $e_{n}(x)=\mathcal{O}\left(q^{\sqrt{n}}\right)$ as $n \rightarrow \infty$, uniformly in $x$ with some (unspecified) $0<q<1$. One year later, using a different method, Paul Lévy [11] improved Kuzmin's result by showing that $\left|e_{n}(x)\right| \leq q^{n}, n \in \mathbb{N}_{+}, x \in I$, with $q=3.5-2 \sqrt{2}=0.67157 \ldots$. The Gauss-Kuzmin-Lévy theorem is the first basic result in the rich metrical theory of continued fractions. Generalizations of these problems for nonregular continued fractions are also called as the Gauss-Kuzmin problem and the Gauss-Kuzmin-Lévy problem [7, 10, 12, 13, 14]. 


\subsection{A non-regular continued fraction expansion}

In this paper we consider another expansion of reals in the unit interval, different from the RCF expansion. In fact, one particular expansion discussed by Adams and Davison in [1], which generalizes the expansion of Davison from [5], has raised to a new type of continued fraction: the digits of the expansion of any number in the unit interval are differences of consecutive non-positive integer powers of an integer $m \geq 2$.

In [7], Iosifescu and Sebe claimed that any $x \in I:=[0,1)$ can be written in the form

$$
x=\frac{m^{-a_{1}(x)}}{1+\frac{m^{-a_{2}(x)}}{1+\frac{m^{-a_{3}(x)}}{1+\ddots}}}:=\left[\left[a_{1}(x), a_{2}(x), a_{3}(x), \ldots\right]\right],
$$

where $m \in \mathbb{N}_{+}, m \geq 2$ and $a_{n}(x)$ 's are integers greater than or equal to -1 .

For any $m \in \mathbb{N}_{+}$with $m \geq 2$, define on $I$ the transformation $\tau_{m}$ by

$$
\tau_{m}(x)=\left\{\begin{array}{lll}
m^{\left\{\frac{\log x^{-1}}{\log m}\right\}}-1, & \text { if } \quad x \neq 0 \\
0, & \text { if } \quad x=0
\end{array}\right.
$$

where $\{\cdot\}$ stands for fractionary part. It is easy to see that $\tau_{m}$ maps the set $\Omega$ of irrationals in $I$ into itself. For any $x \in(0,1)$ put

$$
a_{n}(x)=a_{1}\left(\tau_{m}^{n-1}(x)\right), \quad n \in \mathbb{N}_{+},
$$

with $\tau_{m}^{0}(x)=x$ and

$$
a_{1}(x)= \begin{cases}\left\lfloor\log x^{-1} / \log m\right\rfloor, & \text { if } \quad x \neq 0 \\ \infty, & \text { if } \quad x=0\end{cases}
$$

where $\lfloor\cdot\rfloor$ stands for integer part.

These statements have been proven in [9].

\section{A brief overview of the metrical theory of regular con- tinued fraction expansions}

This section is a short presentation of the regular continued fraction expansion. No proofs are given. They can be found mainly in [6]. 
Denote by $\Omega$ the set of irrationals in the unit interval $I$. Writing $\tau^{n}$ for the $n$th iteration of $\tau$, where $n \in \mathbb{N}:=\{0,1,2,3, \ldots\}$ with $\tau^{0}$ being the identity map, the positive integers

$$
a_{n}(\omega)=a_{1}\left(\tau^{n-1}(\omega)\right), \quad n \in \mathbb{N}_{+},
$$

are the (RCF) digits (also known as partial quotients or incomplete quotients) of $\omega \in \Omega$. Here

$$
a_{1}(\omega)=\lfloor 1 / \omega\rfloor,
$$

and it follows from the definition of $\tau$ and $a_{1}$ that

$$
\omega=\frac{1}{a_{1}(\omega)+\tau(\omega)}, \ldots, \tau^{n}(\omega)=\frac{1}{a_{n+1}(\omega)+\tau^{n+1}(\omega)}, \ldots
$$

Hence

$$
\omega=\frac{1}{a_{1}(\omega)+\frac{1}{a_{2}(\omega)+\ddots+\frac{1}{a_{n}(\omega)+\tau^{n}(\omega)}}}, n \in \mathbb{N}_{+} .
$$

If we set

$$
\left[x_{1}\right]=\frac{1}{x_{1}},\left[x_{1}, x_{2}, \ldots, x_{n}\right]=\frac{1}{x_{1}+\left[x_{2}, x_{3}, \ldots, x_{n}\right]}, n \geq 2,
$$

for arbitrary indeterminates $x_{i}, 1 \leq i \leq n$, then we can write

$$
\omega=\left[a_{1}(\omega), a_{2}(\omega), \ldots, a_{n}(\omega)+\tau^{n}(\omega)\right], n \geq 1,
$$

and one has that, see, e.g., [6],

$$
\omega=\lim _{n \rightarrow \infty}\left[a_{1}(\omega), a_{2}(\omega), \ldots, a_{n}(\omega)\right], \omega \in \Omega .
$$

This last equation will be also written as

$$
\omega=\left[a_{1}(\omega), a_{2}(\omega), \ldots\right], \omega \in \Omega .
$$

The rational numbers $p_{n}(\omega) / q_{n}(\omega)=\left[a_{1}(\omega), a_{2}(\omega), \ldots, a_{n}(\omega)\right]$ are the RCFconvergents of $\omega$. Here we assume that g.c.d. $(p(\omega), q(\omega))=1, n \in \mathbb{N}$. The sequences $\left(p_{n}(\omega)\right)_{n \in \mathbb{N}_{+}}$and $\left(q_{n}(\omega)\right)_{n \in \mathbb{N}_{+}}$satisfy

$$
\begin{array}{lll}
q_{0}(\omega):=1, & q_{n+1}(\omega)=a_{n+1} q_{n}(\omega)+q_{n-1}(\omega), & n \in \mathbb{N}, \\
p_{0}(\omega):=0, & p_{n+1}(\omega)=a_{n+1} p_{n}(\omega)+p_{n-1}(\omega), & n \in \mathbb{N} .
\end{array}
$$

Roughly speaking, the metrical theory of the RCF expansion is about properties of the sequence $\left(a_{n}\right)_{n \in \mathbb{N}_{+}}$ 
The probabilistic structure of the sequence $\left(a_{n}\right)_{n \in \mathbb{N}_{+}}$under $\lambda$ was studied in [6] and is described by the equations

$$
\begin{aligned}
\lambda\left(a_{1}=i\right) & =\frac{1}{i(i+1)}, \quad i \in \mathbb{N}_{+}, \\
\lambda\left(a_{n+1}=i \mid a_{1}, \ldots, a_{n}\right) & =\frac{s_{n}+1}{\left(s_{n}+i\right)\left(s_{n}+i+1\right)}, \quad i, n \in \mathbb{N}_{+},
\end{aligned}
$$

where

$$
s_{n}=\left[a_{n}, \ldots, a_{1}\right] .
$$

These relations follow from the Brodén-Borel-Lévy formula:

$$
\lambda\left(\tau^{n}<x \mid a_{1}, \ldots, a_{n}\right)=\frac{\left(s_{n}+1\right) x}{s_{n} x+1}, \quad x \in I, n \in \mathbb{N}_{+} .
$$

Let $\mathcal{B}_{I}$ denote the $\sigma$-algebra of Borel subsets of $I$. Then the digits $a_{n}, n \in \mathbb{N}_{+}$, are positive integer-valued random variables which are defined almost surely on $\left(I, \mathcal{B}_{I}\right)$ with respect to any probability measure $\mu$ on $\mathcal{B}_{I}$, which assigns probability zero to the set $I \backslash \Omega$ of rationals in $I$. An example of such a measure is Lebesgue measure $\lambda$, but a more important one in the present context is Gauss' measure $\gamma$, which is defined by

$$
\gamma(A)=\frac{1}{\log 2} \int_{A} \frac{\mathrm{d} x}{1+x}, \quad A \in \mathcal{B}_{I}
$$

We see that $\gamma$ is $\tau$-invariant, that is $\gamma(A)=\gamma\left(\tau^{-1}(A)\right), A \in \mathcal{B}_{I}$. Hence, by its very definition, the sequence $\left(a_{n}\right)_{n \in \mathbb{N}_{+}}$is strictly stationary on $\left(I, \mathcal{B}_{I}, \gamma\right)$.

\section{Another continued fraction expansions}

At the concluding remarks in [7], it was stated that if define on $I_{m}:=[0, m-1]$, with $m \in \mathbb{N}, m \geq 3$ the transformation $\tau_{m}$ by

$$
\tau_{m}(x):= \begin{cases}m^{\frac{\log x^{-1}}{\log m}-\left\lfloor\frac{\log x^{-1}}{\log m}\right\rfloor}-1, & \text { if } x \neq 0 \\ 0, & \text { if } x=0\end{cases}
$$

then any real number in $I_{m}$ can be written in the form

$$
\frac{m^{-b_{1}(x)}}{1+\frac{m^{-b_{2}(x)}}{1+\ddots}}:=\left[\left[b_{1}(x), b_{2}(x), \ldots\right]\right],
$$


where $b_{n}=b_{n}(x)$ are integers in $\mathbb{Z}_{\geq-1}:=\{-1,0,1,2,3, \ldots\}$, for any $n \in \mathbb{N}_{+}$, with

$$
b_{1}(x)= \begin{cases}\left\lfloor\log x^{-1} / \log m\right\rfloor, & \text { if } \quad x \neq 0 \\ \infty, & \text { if } \quad x=0\end{cases}
$$

and

$$
b_{n}(x)=b_{1}\left(\tau_{m}^{n-1}(x)\right), \quad n \in \mathbb{N}_{+} .
$$

Remark. Some particular cases of this type of continued fractions have been studied before. For example, by setting $q:=1 / m$ and $b_{n}:=n$, the right-hand side of (12) gives the well-known continued fraction of Rogers and Ramanujan

$$
\frac{q}{1+\frac{q^{2}}{1+\frac{q^{3}}{1+\ddots}}}
$$

Another example is the beautiful result due to Adams and Davison [1]. Let $b_{n}=F_{n}$, where $F_{n}$ is the $n$-th Fibonacci number. Adams and Davison showed that

$$
\frac{m^{-F_{1}}}{1+\frac{m^{-F_{2}}}{1+\frac{m^{-F_{3}}}{1+\ddots}}}=\frac{m-1}{m} \sum_{n \geq 1} m^{-\lfloor n \phi\rfloor}
$$

where $\phi$ is the Golden Ratio.

In [9], the first author presented some metric properties of this continued fraction expansion.

Let $\Omega_{m}$ be the set of all irrational numbers in $I_{m}$. It is easy to check that

$$
\tau_{m}^{n-1}(\omega)=\frac{m^{-b_{n}(\omega)}}{1+\tau_{m}^{n}(\omega)}, \quad \omega \in \Omega_{m}, n \in \mathbb{N}_{+},
$$

hence

$$
\omega=\frac{m^{-b_{1}(\omega)}}{1+\frac{m^{-b_{2}(\omega)}}{1+\cdot \cdot+\frac{m^{-b_{n}(\omega)}}{1+\tau_{m}^{n}(\omega)}}}, n \in \mathbb{N}_{+} .
$$

If we set

$$
\left[\left[x_{1}\right]\right]=m^{-x_{1}}, \quad\left[\left[x_{1}, \ldots, x_{n}\right]\right]=\frac{m^{-x_{1}}}{1+\left[\left[x_{2}, \ldots, x_{n}\right]\right]}, \quad n \geq 2,
$$


for arbitrary indeterminates $x_{i}, 1 \leq i \leq n$, then (17) can be written as

$$
\begin{aligned}
\omega & =\left[\left[b_{1}(\omega)+\frac{\log \left(1+\tau_{m}(\omega)\right)}{\log m}\right]\right]=\left[\left[b_{1}(\omega), b_{2}(\omega)+\frac{\log \left(1+\tau_{m}^{2}(\omega)\right)}{\log m}\right]\right] \\
& =\left[\left[b_{1}(\omega), \ldots, b_{n-1}(\omega), b_{n}(\omega)+\frac{\log \left(1+\tau_{m}^{n}(\omega)\right)}{\log m}\right]\right]
\end{aligned}
$$

where the last equation holds for $n \geq 3$.

We will usually drop the dependence on $\omega$ in the notation. Define

$$
\omega_{0}:=0, \quad \omega_{n}:=\left[\left[b_{1}(\omega), b_{2}(\omega), \ldots, b_{n}(\omega)\right]\right], \quad n \in \mathbb{N}_{+} .
$$

Clearly,

$$
\omega_{n}=\frac{p_{n}}{q_{n}}, \quad n \in \mathbb{N}_{+},
$$

where $p_{n}$ and $q_{n}, n \in \mathbb{N}, n \geq 2$, are integer-valued functions sequences which can be recursively defined on $\Omega_{m}$ by

$$
\begin{aligned}
p_{n} & :=m^{b_{n}} p_{n-1}+m^{b_{n-1}} p_{n-2}, \\
q_{n} & :=m^{b_{n}} q_{n-1}+m^{b_{n-1}} q_{n-2},
\end{aligned}
$$

with $p_{0}:=0, q_{0}:=1, p_{1}:=1$ and $q_{1}:=m^{b_{1}}$. The number $\omega_{n}$ is called the $n$th convergent of $\omega$.

By induction, it is easy to prove that

$$
p_{n} q_{n+1}-p_{n+1} q_{n}=(-1)^{n+1} m^{b_{1}+\ldots+b_{n}}, \quad n \in \mathbb{N}_{+},
$$

and

$$
\frac{m^{-b_{1}}}{1+\frac{m^{-b_{2}}}{1+\ddots+\frac{m^{-b_{n}}}{1+t}}}=\frac{p_{n}+t m^{b_{n}} p_{n-1}}{q_{n}+t m^{b_{n}} q_{n-1}}, \quad n \in \mathbb{N}_{+}, t \geq 0 .
$$

It follows from (18) and (22) that

$$
\omega=\frac{p_{n}(\omega)+\tau_{m}^{n}(\omega) m^{b_{n}(\omega)} p_{n-1}(\omega)}{q_{n}(\omega)+\tau_{m}^{n}(\omega) m^{b_{n}(\omega)} q_{n-1}(\omega)}, \quad \omega \in \Omega_{m}, n \in \mathbb{N}_{+} .
$$

Hence, using (21) we have

$$
\left|\omega-\frac{p_{n}(\omega)}{q_{n}(\omega)}\right|=\frac{\tau_{m}^{n}(\omega) m^{b_{1}(\omega)+\ldots+b_{n}(\omega)}}{q_{n}(\omega)\left(q_{n}(\omega)+\tau_{m}^{n}(\omega) m^{b_{n}(\omega)} q_{n-1}(\omega)\right)},
$$


for any $\omega \in \Omega_{m}$ and $n \in \mathbb{N}_{+}$. Using a similar reasoning as in [7], we have

$$
\left|\omega-\frac{p_{n}(\omega)}{q_{n}(\omega)}\right| \leq \frac{1}{\max \left(F_{n}, m^{b_{1}(\omega)+\ldots+b_{n}(\omega)}\right)},
$$

for any $\omega \in \Omega_{m}$ and $n \in \mathbb{N}_{+}$.

It then appears that

$$
\omega=\lim _{n \rightarrow \infty}\left[\left[b_{1}(\omega), \ldots, b_{n}(\omega)\right]\right], \quad \omega \in \Omega_{m},
$$

which is the precise meaning of $(12)$.

If $i^{(n)}=\left(i_{1}, \ldots, i_{n}\right)$, and $i_{n} \in \mathbb{Z}_{\geq-1}, n \in \mathbb{N}_{+}$then the fundamental interval of rank $n$ corresponding to this type of expansions is defined as

$$
I_{m}\left(i^{(n)}\right)=\left\{\omega \in \Omega_{m}: b_{k}(\omega)=i_{k}, 1 \leq k \leq n\right\},
$$

with the convention that $I_{m}\left(i^{(0)}\right)=\Omega_{m}$.

We will write $I_{m}\left(b_{1}, \ldots, b_{n}\right)=I_{m}\left(b^{(n)}\right), n \in \mathbb{N}_{+}$.

By (23) we have

$$
I_{m}\left(b^{(n)}\right)=\Omega_{m} \cap\left(u\left(b^{(n)}\right), v\left(b^{(n)}\right)\right)
$$

where

$$
u\left(b^{(n)}\right):= \begin{cases}\frac{p_{n}+(m-1) m^{b_{n}} p_{n-1}}{q_{n}+(m-1) m^{b_{n}} q_{n-1}}, & \text { if } n \text { is odd } \\ \frac{p_{n}}{q_{n}}, & \text { if } n \text { is even }\end{cases}
$$

and

$$
v\left(b^{(n)}\right):= \begin{cases}\frac{p_{n}}{q_{n}}, & \text { if } n \text { is odd } \\ \frac{p_{n}+(m-1) m^{b_{n}} p_{n-1}}{q_{n}+(m-1) m^{b_{n}} q_{n-1}}, & \text { if } n \text { is even. }\end{cases}
$$

Let $\mathcal{B}_{I_{m}}$ denote the $\sigma$-algebra of Borel subsets of $I_{m}$. Define the measure $\lambda_{m}$ on $\left(I_{m}, \mathcal{B}_{I_{m}}\right)$ by

$$
\lambda_{m}(A)=\frac{1}{m-1} \cdot \lambda(A), \quad A \in \mathcal{B}_{I_{m}}
$$

where $\lambda$ denotes the Lebesgue measure. Then $\lambda_{m}$ is a probability measure such that $\lambda_{m}\left(I_{m} \backslash \Omega_{m}\right)=0$.

It then follows from (28), (29) and (21) that

$$
\lambda_{m}\left(I_{m}\left(b^{(n)}\right)\right)=\frac{m^{b_{1}+\ldots+b_{n}}}{q_{n}\left(q_{n}+(m-1) m^{b_{n}} q_{n-1}\right)} .
$$




\section{Main theorems}

In this section, we show our main theorems. For this purpose, we define the random variables $\left(s_{n}\right)_{n \in \mathbb{N}_{+}}$by

$$
s_{n}=m^{-b_{n}} \frac{q_{n}}{q_{n-1}}-1, \quad n \in \mathbb{N}_{+},
$$

with $s_{1}=0$. Note that $(20)$ implies that

$$
s_{n}=\frac{m^{-b_{n}}}{1+s_{n-1}}, n \in \mathbb{N}_{+}, \text {with } s_{1}=0 .
$$

Hence

$$
s_{n}=\frac{m^{-b_{n}}}{1+\frac{m^{-b_{n-1}}}{1+\cdot+\frac{m^{-b_{3}}}{1+m^{-b_{2}}}}}=\left[\left[b_{n}, b_{n-1}, \ldots, b_{2}, \infty\right]\right], n \geq 2 .
$$

In the metrical theory of continued fractions, the Brodén-Borel-Lévy formula is known as a nontrivial probabilistic property of the dynamical system arising from regular continued fraction expansions on the unit interval $[0,1]$. We give now the Brodén-Borel-Lévy type formula associated with the continued fraction expansions in (12).

Theorem 1 (Brodén-Borel-Lévy type formula). For any $n \in \mathbb{N}_{+}$we have

$$
\lambda_{m}\left(\tau_{m}^{n}<x \mid b_{1}, \ldots, b_{n}\right)=\frac{\left(s_{n}+m\right) x}{(m-1)\left(s_{n}+x+1\right)}, x \in I_{m},
$$

where $s_{n}$ is defined by (32) or (33).

The equation (35) is the Brodén-Borel-Lévy formula for these continued fraction expansions and allows us to determine the probability structure of $\left(b_{n}\right)_{n \in \mathbb{N}_{+}}$under $\lambda_{m}$.

Proposition 2. For any $i \in \mathbb{Z}_{\geq-1}$ and $n \in \mathbb{N}_{+}$we have

$$
\lambda_{m}\left(b_{1}=i\right)=m^{-(i+1)}
$$

and

$$
\lambda_{m}\left(b_{n+1}=i \mid b_{1}, \ldots, b_{n}\right)=P_{i}\left(s_{n}\right),
$$

where

$$
P_{-1}\left(s_{n}\right)=1-\frac{s_{n}+m}{(m-1)\left(s_{n}+2\right)}
$$


and for $i \in \mathbb{N}$

$$
P_{i}\left(s_{n}\right)=\frac{m^{-(i+1)}\left(s_{n}+1\right)\left(s_{n}+m\right)}{\left(s_{n}+m^{-i}+1\right)\left(s_{n}+m^{-(i+1)}+1\right)} .
$$

Now, since

$\frac{m^{-(i+1)}(x+1)(x+m)}{\left(x+m^{-i}+1\right)\left(x+m^{-(i+1)}+1\right)}=\frac{1}{m-1}\left(\frac{1}{x+m^{-(i+1)}+1}-\frac{1}{x+m^{-i}+1}\right)$,

then

$$
\sum_{i \geq-1} P_{i}(x)=1, \text { for all } x \in I_{m} .
$$

Thus, the function $P_{i}(x)$ defines a transition probability function from $\left(I_{m}, \mathcal{B}_{I_{m}}\right)$ to $(\mathbb{N}, \mathcal{P}(\mathbb{N}))$.

Corollary 3. The Proposition 2 shows that $\left(s_{n}\right)_{n \in \mathbb{N}_{+}}$with $s_{1}=0$ is a homogeneous $I_{m}$-valued Markov chain on $\left(I_{m}, \mathcal{B}_{I_{m}}, \lambda_{m}\right)$, with the following transition mechanism: from state $s \in I_{m} \backslash \Omega_{m}, s \geq 1$ the only possible one-step transitions are those to states $m^{-i} /(s+1), i \in \mathbb{Z}_{\geq-1}$, with corresponding probabilities $P_{i}(s), i \in \mathbb{Z}_{\geq-1}$.

At this point, we question whether the invariant measure of the transformation $\tau_{m}$ exists on $\mathcal{B}_{I_{m}}$. Adler's folklore theorem (see, e.g., [2]) clearly show the existence of such a measure. Another interesting way to show that is the following theorem.

Theorem 4. Let $m \geq 2$ and let $\mathcal{B}_{I_{m}}$ denote the $\sigma$-algebra of Borel subsets of $I_{m}$. If $\tau$ is the Gauss measure and $\tau_{m}$ are the transformations from (11), then there exists a $\tau_{m}$-invariant measure on $\mathcal{B}_{I_{m}}$ such that $\tau$ and $\tau_{m}$ are conjugate by the measure preserving map.

Remark. The explicit expression of the invariant measure of the transformation $\tau_{m}$ remains an open problem.

\section{Proofs of the main theorems}

In this section, we prove main theorems.

Proof of Theorem 1. Clearly, for any $n \in \mathbb{N}_{+}$

$$
\lambda_{m}\left(\tau_{m}^{n}<x \mid b_{1}, \ldots, b_{n}\right)=\frac{\lambda\left(\left(\tau_{m}^{n}<x\right) \cap I\left(b_{1}, \ldots, b_{n}\right)\right)}{\lambda_{m}\left(I\left(b_{1}, \ldots, b_{n}\right)\right)} .
$$


From (21) and (27) we have

$$
\begin{aligned}
\lambda_{m}\left(\left(\tau_{m}^{n}<x\right) \cap I\left(b_{1}, \ldots, b_{n}\right)\right) & =\frac{1}{m-1}\left|\frac{p_{n}}{q_{n}}-\frac{p_{n}+x m^{b_{n}} p_{n-1}}{q_{n}+x m^{b_{n}} q_{n-1}}\right| \\
& =\frac{1}{m-1} \frac{x m^{b_{1}+\ldots+b_{n}}}{q_{n}\left(q_{n}+x m^{b_{n}} q_{n-1}\right)} .
\end{aligned}
$$

Hence, using (31) and (32),

$$
\begin{aligned}
\lambda_{m}\left(\tau_{m}^{n}<x \mid b_{1}, \ldots, b_{n}\right) & =\frac{1}{m-1} \frac{x\left(q_{n}+(m-1) m^{b_{n}} q_{n-1}\right)}{q_{n}+x m^{b_{n}} q_{n-1}} \\
& =\frac{\left(s_{n}+m\right) x}{(m-1)\left(s_{n}+x+1\right)}
\end{aligned}
$$

for any $x \in I_{m}$ and $n \in \mathbb{N}_{+}$.

Proof of Proposition 2. From (26), we have

$$
\left\{\omega \in \Omega_{m}: b_{1}(\omega)=i\right\}=\Omega_{m} \cap\left(\frac{1}{m^{i+1}}, \frac{1}{m^{i}}\right) .
$$

Thus

$$
\lambda_{m}\left(b_{1}=i\right)=\frac{1}{m-1}\left|\frac{1}{m^{i+1}}-\frac{1}{m^{i}}\right|=m^{-(i+1)} .
$$

From (16), we have

$$
\tau_{m}^{n}(\omega)=\left[\left[b_{n+1}, b_{n+2}, \ldots\right]\right], n \in \mathbb{N}_{+}, \omega \in \Omega_{m} .
$$

Then, for $i=-1$, using (35), we have

$$
\begin{aligned}
\lambda_{m}\left(b_{n+1}=i \mid b_{1}, \ldots, b_{n}\right) & =\lambda_{m}\left(\tau_{m}^{n} \in(1, m-1] \mid b_{1}, \ldots, b_{n}\right) \\
& =\lambda_{m}\left(\tau_{m}^{n}<m-1\right)-\lambda_{m}\left(\tau_{m}^{n}<1\right) \\
& =1-\frac{s_{n}+m}{(m-1)\left(s_{n}+2\right)} .
\end{aligned}
$$


For $i \in \mathbb{N}$, we have

$$
\begin{aligned}
\lambda_{m}\left(b_{n+1}=i \mid b_{1}, \ldots, b_{n}\right) & =\lambda_{m}\left(\tau_{m}^{n} \in\left(\frac{1}{m^{i+1}}, \frac{1}{m^{i}}\right] \mid b_{1}, \ldots, b_{n}\right) \\
& =\lambda_{m}\left(\tau_{m}^{n}<\frac{1}{m^{i}}\right)-\lambda_{m}\left(\tau_{m}^{n}<\frac{1}{m^{i+1}}\right) \\
& =\frac{\left(s_{n}+m\right) m^{-i}}{(m-1)\left(s_{n}+m^{-i}+1\right)} \\
& -\frac{\left(s_{n}+m\right) m^{-(i+1)}}{(m-1)\left(s_{n}+m^{-(i+1)}+1\right)} \\
& =\frac{m^{-(i+1)}\left(s_{n}+1\right)\left(s_{n}+m\right)}{\left(s_{n}+m^{-i}+1\right)\left(s_{n}+m^{-(i+1)}+1\right)} .
\end{aligned}
$$

Proof of Theorem 4. Let $\Omega$ and $\Omega_{m}$ denote the irrational numbers in $I$ and $I_{m}$, respectively, and

$$
\begin{aligned}
\mathbb{N}_{+}^{\infty} & :=\left\{\left(l_{i}\right)_{i \in \mathbb{N}_{+}}: l_{i} \in \mathbb{N}_{+} \text {for } i \in \mathbb{N}_{+}\right\} \\
\mathbb{Z}_{\geq-1}^{\infty}: & :=\left\{\left(l_{i}\right)_{i \in \mathbb{N}_{+}}: l_{i} \in \mathbb{Z}_{\geq-1} \text { for } i \in \mathbb{N}_{+}\right\} .
\end{aligned}
$$

By using the continued fraction expansions associated with $\tau_{m}$, we obtain the following set-theoretical bijections

$$
\Omega \cong \mathbb{N}_{+}^{\infty} \text { and } \Omega_{m} \cong \mathbb{Z}_{\geq-1}^{\infty},
$$

such that

$$
\begin{aligned}
\Omega \ni x & \mapsto\left(a_{n}(x)\right)_{n \in \mathbb{N}_{+}} \in \mathbb{N}_{+}^{\infty}, \\
\Omega_{m} \ni x & \mapsto\left(b_{n}(x)\right)_{n \in \mathbb{N}_{+}} \in \mathbb{Z}_{\geq-1}^{\infty},
\end{aligned}
$$

with $a_{1}, a_{2}, \ldots$ defined by (4) and (5) and $b_{1}, b_{2}, \ldots$ defined by (13) and (14). From these, we can define the bijective map $\theta_{m}$ from $\Omega$ to $\Omega_{m}$ by

$$
\theta_{m}(x):=\left[\left[a_{1}(x)-2, a_{2}(x)-2, a_{3}(x)-2, \ldots\right]\right], \quad x \in \Omega .
$$

In consequence, we obtain the set-theoretical conjugate map $\theta_{m}$ between $\tau$ and $\tau_{m}$, i.e.,

$$
\theta_{m} \circ \tau \circ \theta_{m}^{-1}=\tau_{m} .
$$

By the definitions of $\Omega$ and $\Omega_{m}$, (44) holds on $I_{m}$ except for a set of Lebesgue measure zero. Using $\theta_{m}$, we construct the invariant measure of $\tau_{m}$ denoted by $\gamma_{m}$ as

$$
\gamma_{m}=\gamma \circ \theta_{m}^{-1}
$$


Then

$$
\gamma_{m}\left(\tau_{m}^{-1}(E)\right)=\gamma\left(\left(\tau^{-1} \circ \theta_{m}^{-1}\right)(E)\right)=\gamma_{m}(E),
$$

for each $E \in \mathcal{B}_{I_{m}}$.

Remark. Theorem 4 is proved by the construction of the conjugate map $\theta_{m}$ from the following diagram of four dynamical systems with invariant measures:

$$
\begin{array}{lll}
(\Omega, \tau, \gamma) & \stackrel{\theta_{m}}{\cong} & \left(\Omega_{m}, \tau_{m}, \gamma_{m}\right) \\
2 \| & \circlearrowleft & 2 \| \\
\left(\mathbb{N}_{+}^{\infty}, \hat{\tau}, \hat{\gamma}\right) & \cong & \left(\mathbb{Z}_{\geq-1}^{\infty}, \hat{\tau}_{m}, \hat{\gamma}_{m}\right)
\end{array}
$$

where $m \geq 2$, and $\left(\mathbb{N}_{+}^{\infty}, \hat{\tau}, \hat{\gamma}\right)$ and $\left(\mathbb{Z}_{>-1}^{\infty}, \hat{\tau}_{m}, \hat{\gamma}_{m}\right)$ are dynamical systems with standard shift transformations $\hat{\tau}$ and $\hat{\tau}_{m}$, and their invariant measures $\hat{\gamma}$ and $\hat{\gamma}_{m}$ induced from $(\Omega, \tau, \gamma)$, respectively.

\section{References}

[1] Adams, W.W. and Davison, J.L., A remarkable class of continued fractions, Proc. Amer. Math. Soc. 65 (1977) 194-198.

[2] Boyarsky, A. and Góra, P., Laws of Chaos: Invariant Measures and Dynamical Systems in One Dimension, Birkhäuser, Boston, 1997.

[3] Brezinski, C., History of Continued Fractions and Padé Approximants. Springer Series in Computational Mathematics 12, Springer-Verlag, Berlin, 1991.

[4] Corless, R.M., Continued fractions and chaos, Amer. Math. Monthly 99(3) (1992), 203-215.

[5] Davison, J.L., A series and its associated continued fraction, Proc. Amer.Math. Soc.63(1) (1977) 29-32.

[6] Iosifescu, M. and Kraaikamp C., Metrical theory of continued fractions, Kluwer Academic, 2002. 
[7] Iosifescu, M. and Sebe, G.I., An exact convergence rate in a GaussKuzmin-Lévy problem for some continued fraction expansion, in vol. Mathematical Analysis and Applications, 90-109. AIP Conf. Proc. 835 (2006), Amer.Inst.Physics, Melville, NY.

[8] Kuzmin, R.O., On a problem of Gauss. Dokl. Akad. Nauk SSSR Ser. A (1928) 375-380. [Russian; French version in Atti Congr. Internaz.Mat. (Bologna, 1928), Tomo VI, pp.83-89. Zanichelli, Bologna, 1932].

[9] Lascu, D., Markov processes in probabilistic number theory, Ph.D. thesis, Romanian Academy, 2010. (Romanian)

[10] Lascu, D., On a Gauss-Kuzmin-type problem for a family of continued fraction expansions, J. Number Theory 133(7) (2013), 2153-2181.

[11] Lévy, P., Sur les lois de probabilité dont dépendent les quotients complets et incomplets d'une fraction continue. Bull. Soc. Math. France $\mathbf{5 7}$ (1929) 178-194.

[12] Sebe, G.I., On convergence rate in the Gauss-Kuzmin problem for grotesque continued fractions, Monatsh. Math. 133 (2001), 241-254.

[13] Sebe, G.I., A Gauss-Kuzmin theorem for the Rosen fractions, J.Théor.Nombres Bordx. 14 (2002), 667-682.

[14] Sebe, G.I. and Lascu, D. A Gauss-Kuzmin theorem and related questions for theta-expansions, arXiv preprint arXiv:1305.5563 (2013), 1-27.

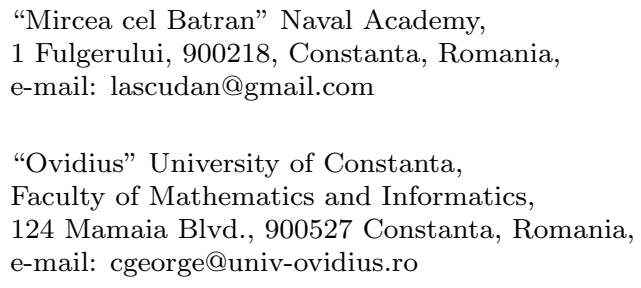

